\title{
Los Estudios de Impacto Ambiental y su Implicancia en las Inversiones de los Proyectos
}

RESUMEN

Los Proyectos de inversión al intervenir en distintos medios generan un conjunto de impactos, ya sean propios del proyecto como también en los aspectos sociales y ambientales, los que se deben incorporar en la evaluación para determinar su viabilidad.El artículo tiene como objetivo análizar el desarrollo de los estudios de impacto ambiental en relación a los proyectos y proponer un enfoque de integración entre ambos.

Palabras Claves: Proyecto de inversión, impactos, estudio de impacto ambiental

\section{ENVIRONMENTAL IMPACT STUDIES AND ITS IMPLICATION ON THE PROJECT INVESTMENTS}

\section{ABSTRACT}

The investment projects involved various means to generate a set of impacts, whether they own the project, social and environmental issues that should be incorporated into the evaluation to determine its viability. The following article has got the principal objetive of analyze the development of environmental impact estudies in relation to the projects and propose a focus on integration between both.

Keywords: Investment project, impacts, environmental impact study

\section{INTRODUCCIÓN}

En los últimos años en el país se han desarrollado una mayor cantidad de proyectos de inversión de distinta naturaleza, siendo este un factor positivo generador de desarrollo regional y local. Pero en muchos casos dichas intervenciones pueden generar impactos negativos en el ambiente, por lo que se exige según regulación normativa tanto para el sector público como para el privado, realizar los estudios de impacto ambiental para identificar los posibles impactos negativos y las acciones ha realizar para evitarlos.

Los estudios de impacto ambiental se sustentan en un conjunto de metodologias y herramientas, los que se deben integrar al análisis del proyecto.

El presente artículo se sustenta en el análisis de un conjunto de estudios de impacto ambiental desarrollados para determinar su estructura y enfoque metodológico adecuado en relación a los impactos, además de brindar un enfoque conceptual que lo integre a la evaluación de proyectos.

\section{PROYECTO DE INVERSIÓN (1)}

El proyecto de inversión desde una perspectiva general, se entiende como una intervención en un medio para dar solución a una problemática existente y lograr un cambio deseado, por lo que va a existir un grupo de personas involucradas y necesidades referidas a la problemática.

El enfoque de desarrollo de un proyecto de inversión se inicia con la identificación del problema, luego se cuantifica, en función a ello se determina los requerimientos, cuya valorización se convierte en la inversión del proyecto, esquema que se muestra en la figura $\mathrm{N}^{\circ} 1$.

\section{Figura $\mathbf{N}^{0} 1$}

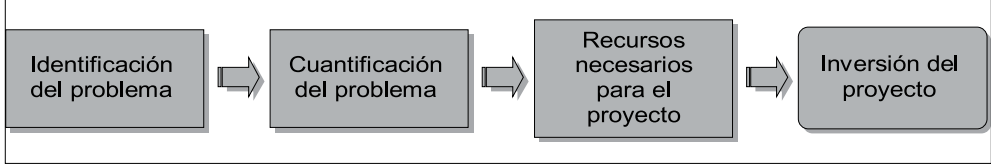

Fuente: Elaboración Propia

Magíster en Gestión Económica y Empresarial, Ingeniero Industrial UNMSM, Profesor en la Facultad de Ingeniería Industrial, Departamento Académico de Producción y Gestión Industrial de la UNMSM.

Email: cieandia@hotmail.com 


\section{Ambiente}

Entiéndase, que toda mención hecha al «ambiente» o a «sus componentes» comprende a los elementos físicos, químicos y biológicos de origen natural o antropogénico que, en forma individual o asociada, conforman el medio en el que se desarrolla la vida, siendo los factores que aseguran la salud individual y colectiva de las personas, la conservación de los recursos naturales, la diversidad biológica y el patrimonio cultural asociado a ellos, entre otros.

\section{SISTEMA NACIONAL DE EVALUACIÓN DEL IMPACTO AMBIENTAL}

El Sistema Nacional de Evaluación del Impacto Ambiental (SEIA) (2), se crea en abril del 2001 como un sistema único y coordinado de identificación, prevención, supervisión, control y corrección anticipada de los impactos ambientales negativos derivados de las acciones humanas.

El Ministerio del Ambiente - MINAM, es el encargado de dirigir y administrar el SEIA

\section{Ámbito de Aplicación del SEIA}

Quedan comprendidos en el ámbito de aplicación de la Ley $N^{\circ} 27446$ las políticas, planes y programas de nivel nacional, regional y local que puedan originar implicaciones ambientales significativas; así como los proyectos de inversión pública, privada o de capital mixto, que impliquen actividades, construcciones, obras y otras actividades comerciales y de servicios que puedan causar impacto ambientales negativos significativos.

\section{Certificación Ambiental}

Toda persona natural o jurídica, de derecho público o privado, nacional o extranjera, que pretenda desarrollar un proyecto de inversión susceptible de generar impactos ambientales negativos de carácter significativo, debe gestionar una Certificación Ambiental ante la autoridad competente que corresponda.

\section{Estudio de Impacto Ambiental}

Se define como un estudio de evaluación, descripción y determinación de impactos de los aspectos físicos, químicos, biológicos, sociales, económicos y culturales en el área de influencia del proyecto, con la finalidad de determinar las condiciones existentes y capacidades del entorno, analizar el ecosistema y prever los riesgos directos e indirectos, indicando las medidas de prevención y las de control.

\section{Plan de Manejo Ambiental}

Forma parte del Estudio de Impacto Ambiental, contiene las medidas de mitigación y compensación, la evaluación e riesgos, las medidas de contingencia y el seguimiento de los impactos.

\section{Situación Actual de los Estudios de Impacto Ambiental}

Los estudios de impacto ambiental se desarrollan bajo una estructura establecida por los sectores correspondientes, en donde uno de los aspectos importantes es la identificación de impactos. Para ello utilizan un conjunto de matrices con características definidas.

Se ha analizado once estudios de impacto ambiental, ellas son:

1. Central Térmica el Faro, año 2011.

2. Compañía Minera Antamina S.A, año 1998.

3. Proyecto Alto Chicama - Minera Barrick Misquichilca S.A., año 2003.

4. Ampliación de la Concentradora Toquepala y Recrecimiento del Embalse de Relaves de quebrada Honda.

5. Proyecto Minero Tía María de Southern Peru Copper Corporation

6. Proyecto La Zanja de la Minera La Zanja S.R.L.

7. Prospección Sísmica 2D y Perforación Exploratoria, lote 138

8. Prospección Sísmica 2D - 3D Y Perforación de 23 pozos exploratorios en Kinteroni, Mapiy Mashira, lote 57"

9. Estudio de impacto ambiental de Proyecto Central Hidroeléctrica San Gabán IV

10. Estudio de Impacto Ambiental de la Línea de Conducción de gas en el tramo Mipaya - Pagorenia del lote 56 .

11. Estudio de Impacto Ambiental para la Línea de Transmisión en 138 kv. S.e. Trujillo nortenueva s.e. Trujillo noroeste, incluida la nueva Subestación

En dichos estudios se ha encontrado generalmente la siguiente información en cuanto a la identificación de impactos en los factores económicos y sociales: 


\begin{tabular}{|c|c|}
\hline Impactos negativos económicos y sociales & Impactos positivos económicos y sociales \\
\hline $\begin{array}{l}\text { - } \quad \text { Mayor tránsito vehicular } \\
\text { - } \quad \text { Mayor número de accidentes viales } \\
\text { - } \quad \text { Pérdida de tierras agrícolas y de pastoreo }\end{array}$ & $\begin{array}{ll}\text { - } & \text { Mejoras en los centros educativos y de salud } \\
\text { - } & \text { Sojora a nivel de infraestructura y servicios } \\
\text { - } & \text { Generación de empleo } \\
\text { - } & \text { Dinamización del comercio y servicios } \\
\text { - } & \text { Oportunidades de desarrollo económico y social } \\
\text { - } & \text { Aumena la Comunidad } \\
\text { - } & \text { Aumento del turismo }\end{array}$ \\
\hline
\end{tabular}

Lo cual indica que los estudios de impacto ambiental incluyen como impactos no solamente los ambientales, sino a todos. Ello puede llevar a duplicidades porque en la evaluación de los proyectos también se incluyen algunos de ellos. Además, la generación de empleo es un "impacto" que se encuentra en todos los estudios, siendo incorrecto porque su valoración está incluida en todas las actividades e impactos desarrollados por el proyecto de inversión.

Así también, del análisis de la información de valoración de los impactos se tiene lo siguiente de una muestra de 11 proyectos analizados:

- El $100 \%$ de los estudios presentan valoración de los Impactos Ambientales del proyecto; de los cuales el $82 \%$ realiza una valoración cualitativa mientras que sólo el $18 \%$ presenta una valoración económica.

- Sólo el 18\% presenta valoración económica del Plan de Manejo Ambiental del proyecto.

- $\quad$ El $27 \%$ de los estudios presenta la valoración cualitativa del Análisis de Costo Beneficio; mientras que el $73 \%$, no valora cualitativa ni cuantitativamente.

Dicha información conduce a determinar que no se presenta un enfoque de valoración económica de los impactos y de los planes de manejo ambiental, por lo que se concluye que los estudios de impactos ambientales son realizados sin integrarse a los proyectos de inversión y su aprobación se da como un documento independiente por ello se duplican todos los impactos. Ello se verifica cuando en los estudios de impacto ambiental incorporan el "análisis beneficio costo" (6) que solo debe realizarse en los proyectos de inversión; por naturaleza el enfoque de un EsIA no es demostrar que los beneficios del proyecto compensan a los impactos negativos.

Así también, los planes de manejo ambiental incorporan todas las acciones para reducir, evitar o compensar los impactos negativos, los que generan un costo; ¿quién lo asume?, por lógica el proyecto, entonces dicho costo debe ser parte de la inversión.

\section{Propuesta Metodológica para los EsIA(3)}

Para lograr una adecuada evaluación del proyecto es necesario identificar correctamente los impactos e incluir los resultados económicos del estudio, por lo que se recomienda modificar el enfoque usual, por uno que integre los proyectos de inversión y los estudios de impacto ambiental.

En primer lugar se debe agrupar los impactos según dimensiones para no duplicarlos; para ello se ha dividido en tres dimensiones: intrínseca, ambiental y social. El siguiente esquema resume la propuesta metodológica apropiada:

Figura N. ${ }^{\circ} 2$

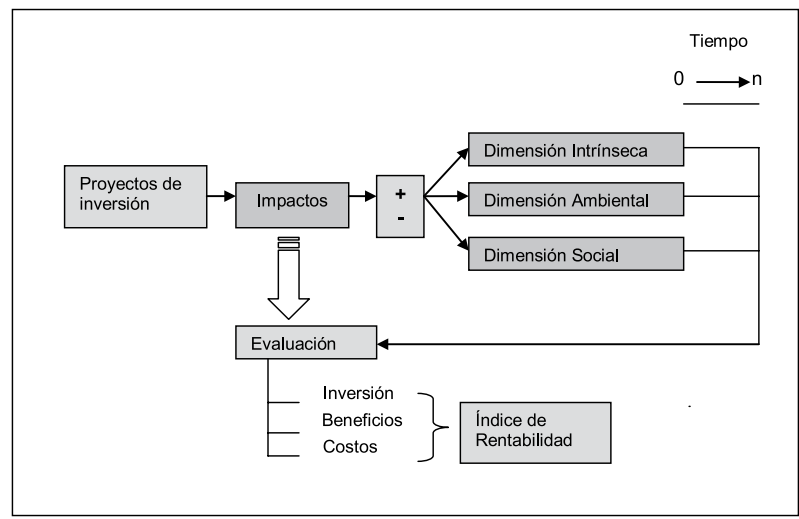

Fuente: Elaboración Propia

La dimensión intrínseca agrupa a los impactos de la naturaleza del proyecto o asociados a su objetivo, por ejemplo, para una carretera los beneficios son los ahorros de costos.

La dimensión ambiental debe agrupar a todos los impactos relacionados exclusivamente al medio ambiente en sí, para ello se elabora el estudio de impacto ambiental.

La dimensión social agrupa a todos los impactos relacionados a los cambios en las costumbres o forma de vida de la población, también es recomendable elaborar un estudio de impacto social.

Bajo está perspectiva no se presentaría duplicidad en la evaluación de impactos; es decir no tiene sen- 
tido que en el EsIA se incorporen impactos de otras dimensiones; los costos y beneficios asociados a cada dimensión se integran al proyecto y el análisis beneficio costo solo se realizaría en la evaluación de proyecto.

\section{CONCLUSIONES}

1. No existe una relación entre la valoración resultado de los estudios de impacto ambiental y la evaluación de los proyectos de inversión que lo originan.

2. Es necesario modificar el enfoque metodológico de los estudios de impacto ambiental por la propuesta que integre dichos estudios con los proyectos de inversión.

3. No se tiene la cultura de valorización económica de los planes de manejo ambiental e impac- tos en los estudios de impacto ambiental para incluirlos en la evaluación de los proyectos de inversión.

\section{REFERENCIAS BIBLIOGRÁFICAS}

[1] Andía Valencia, W. (2010). Gerencia de Proyectos Sociales y de Inversión Social. 1ra edición. El Saber Editores. Perú.

[2] Andía Valencia, W., (2010). Manual de Gestión Ambiental. 2da. edición. El Saber Editores. Perú.

[3] Andía Valencia, W., (2012). Manual de Proyectos de Inversión para el Sector Público. 2da. edición. El Saber Editores. Perú.

[4] Ministerio de Economia y Finanzas, Manuales Metodológicos. 